\title{
Molecular Dynamics Simulation Study of Mechanical Effects of Lubrication on a Nanoscale Contact Process
}

\author{
S. Stephan • M. P. Lautenschlaeger • I. \\ Alabd Alhafez . M. T. Horsch . H. M. \\ Urbassek • H. Hasse
}

Received: date / Accepted: date

\begin{abstract}
Using molecular dynamics simulation, we study the effect of a lubricant on indentation and scratching of a Fe surface. By comparing a dry reference case with two lubricated contacts - differing in the adsorption strength of the lubricant - the effects of the lubricant can be identified. We find that after an initial phase, in which the lubricant is squeezed out of the contact zone, the contact between the indenter and the substrate is essentially dry. The number of lubricant molecules confined in the tip-substrate gap increases with the lubricant adsorption energy. Trapped lubricant broadens the tip area active in the scratching process - mainly on the flanks of the groove - compared to a dry reference case. This leads to a slight increase in chip height and volume, and also contributes to the scratching forces.
\end{abstract}

Keywords nanoindentation · lubrication $\cdot$ molecular dynamics $\cdot$ single asperity contact $\cdot$ squeeze-out

\section{Introduction}

The small zone in which two solid bodies interact directly in a contact process is highly important, e.g. for high precision machining and tribological processes. Unfortunately, the nanoscopic contact zone is practically inaccessible by experiment so that only little is known on the phenomena occurring in that zone. Molecular dynamics simulations are attractive for providing such information.

Dry nanoindentation and dry scratching processes have been studied in detail with different focuses $[1,2]$. For example there are studies on the influence of the shape of the tool [3,4], substrate materials [5-7], indentation depth [6], cutting direction in relation to the lattice orientation [8], grain boundaries [9], surface roughness [10], alloys [11], coatings, [12] and temperature [13].

S. Stephan · M. P. Lautenschlaeger · M. T. Horsch · H. Hasse

TU Kaiserslautern (TUK), Laboratory of Engineering Thermodynamics (LTD)

E-mail: simon.stephan@mv.uni-kl.de

I. Alabd Alhafez · H. M. Urbassek

TU Kaiserslautern (TUK), Physics Department and Research Center OPTIMAS 
For an introduction into lubricated contact processes in general, we refer to the literature [14-17]. The influence of different variables on lubricated contact processes has been studied on the atomistic scale: chain length of the lubricant molecules [18,19], surface roughness [20], adsorbates [21-30], and surface chemistry [31]. There have also been attempts to link atomistic simulation with experiments in that field [32-35]. In most of these studies, lubrication was only considered by including adsorbed layers of fluid molecules on the substrate in the simulation, while studies in which the contact is truly immersed in a liquid are rare [36-38]. An interesting finding is the squeeze-out of the lubricant from the contact zone which has been observed both in simulations in which the lubricant was only a thin adsorbed fluid layer $[39,20]$, as well as in previous works from our group in which the indenter was immersed in the lubricant $[37,38]$. In some studies, the solids were modeled by only a few atom layers [39,20]. Cross-interactions resulting from the simultaneous presence of the bulk fluid phase and the bulk substrate have to the best of our knowledge only been investigated by Rentsch et al. [36] and in previous studies of our group [37,38]. Also the influence of the solid-fluid interaction energy on the lubricated nanoscopic contact process has not yet been investigated systematically.

In the present study, we report on molecular dynamics simulations of a threestage nanoscopic contact process consisting of indentation, scratching and retraction of an indenter on a substrate. The contact process is set up by an iron-like solid substrate, a rigid spherical indenter and a purely dispersively interacting fluid methane modeled by a Lennard-Jones potential. The Lennard-Jones potential [40] is used here to investigate the influence of a simple fluid and due to its generic importance in soft matter physics. Also, liquid methane in contact processes is important for technical applications, e.g. in cryogenic pumps for LNG (liquid natrual gas) processes [41-44] and the next generation of rocket engines $[45,46]$. Furthermore the simple Lennard-Jones fluid is a realistic model for liquid nitrogen $\left(\mathrm{N}_{2}\right)$ [47], which has become an important cutting fluid for cooling in cryogenic machining processes $[48,49]$.

Three cases of the contact process are compared: a dry reference case and two lubricated cases in which the indenter is fully immersed in the liquid lubricant. The two cases differ in the solid-fluid interaction energy, which determines the adsorption strength of the lubricant on the indenter and substrate surface. Becker et al. $[50,51]$ investigated how, for Lennard-Jones systems, the solid-fluid interaction energy correlates with the wetting state, e.g. the contact angle formed between a liquid droplet on a solid surface. The solid-fluid interaction energy is not set here such as to match the actual contact angle of liquid methane on pure iron, which is not known for atomistically flat single crystals. Rather, it is chosen to obtain a fully wetted state (strong attraction) and a state with a finite contact angle of about $71^{\circ}$ (weak attraction).

The influence of the lubrication on various properties of the contact process is examined in this work: normal and tangential forces and the coefficient of friction $(\mathrm{COF})$, chip formation, roughness of the created groove surface, and development of dislocations in the substrate. It is found that after a simulation time in which the lubricant is squeezed out, the contact between the indenter and the substrate is essentially dry. Nevertheless, effects of lubrication are present and depend on the solid-fluid interaction energy. 
The article is organised in two parts: in the first part, the potential model, simulation method and scenario are introduced and observation variables are defined. In the second part the results are presented and discussed.

\section{Methods}

\subsection{Simulation Setup}

Fig. 1 shows the simulation setup. It consists of the rigid spherical indenter with a radius of $R=5 \mathrm{~nm}$, the solid substrate, and in two simulations a lubricant into which the indenter and the substrate are immersed. The size of the substrate block is $52.5 \mathrm{~nm}, 62.6 \mathrm{~nm}, 27.2 \mathrm{~nm}$ in $x$-, $y$ - and $z$-direction, respectively. The substrate surface is in the $x y$-plane. The indenter conducts three sequential movements: (i) indentation, during which the indenter penetrates into the substrate in $-z$ direction normal to the surface, (ii) scratching, during which the indenter moves in $+x$-direction through the substrate forming a groove, and (iii) retraction in $+z$-direction. The movement of the indenter is prescribed, its velocity is $20 \mathrm{~m} / \mathrm{s}$ throughout all three phases of its movement (i)-(iii). The indentation depth is $3 \mathrm{~nm}$ and kept constant during the scratching phase (ii). The origin of the Cartesian coordinate system is defined as follows: $z=0$ for the initial unpenetrated surface of the substrate. $x=0$ and $y=0$ for the initial position of the center of the indenter.

The substrate is fixed in the box by fixing three layers of substrate atoms next to the box margins in $x$ - and $y$-direction and at the bottom. In $x$ - and $y$ direction periodic boundary conditions are applied. The simulation box is confined at the top at $z_{\text {wall }}=28.2 \mathrm{~nm}$ by a soft repulsive wall. The initial temperature is set to $100 \mathrm{~K}$, which is above the melting temperature of the lubricant. This temperature is imposed to the entire system during the equilibration. Furthermore this temperature is imposed to four atom layers between the substrate and the fixed layers, as indicated as thermostat layer in Fig. 1. The temperature of this layer is imposed by velocity scaling [52] throughout the indentation and scratching simulation. The latter temperature controlling extracts dissipated energy from the simulation box.

The fixed thermostated layers of the substrate end 8 atom layers below the substrate surface. The number of atoms is about $9.2 \cdot 10^{4}$ for the indenter, $1.5 \cdot 10^{6}$ for the lubricant, and $7.7 \cdot 10^{6}$ for the substrate.

\subsection{Molecular Model}

Table 1 shows the symmetric interaction matrix of all three substances (substrate, indenter and lubricant) and their cross-interactions. The diagonal of the matrix indicates the interactions within each substance. The off-diagonal indicate unlike interactions.

The substrate is a bcc iron single crystal. Initially, its surface is atomically flat. The substrate-substrate interaction is described by the iron embedded atom model (EAM) of Mendelev et al. [53]. The crystal has a (100) surface; the scratching is performed in $\left[\begin{array}{lll}0 & \overline{1} & \overline{1}\end{array}\right]$ direction, which is aligned with the $x$-axis. 
The indenter atoms are placed on a diamond lattice with a lattice constant of $0.35667 \mathrm{~nm}$. The indenter is a rigid sphere with radius $R=5 \mathrm{~nm}$ with fully prescribed velocities to move the indenter in the respective simulation phase (i)(iii).

The lubricant-lubricant interaction is modeled by single Lennard-Jones truncated and shifted (LJTS) [54] interaction sites, cf. LJTS-lubricant in Table 1. The LJTS potential is defined by [40]

$$
\begin{array}{r}
u_{\mathrm{LJ}}(r)=4 \varepsilon\left[\left(\frac{\sigma}{r}\right)^{12}-\left(\frac{\sigma}{r}\right)^{6}\right] \quad \text { and } \\
u_{\mathrm{LJTS}}(r)= \begin{cases}u_{\mathrm{LJ}}(r)-u_{\mathrm{LJ}}\left(r_{\mathrm{c}}\right) & r \leq r_{\mathrm{C}} \\
0 & r>r_{\mathrm{c}} .\end{cases}
\end{array}
$$

with $u_{\mathrm{LJ}}$ as the full Lennard-Jones potential, $\varepsilon$ and $\sigma$ being the energy and size parameter. The distance between two particles is denoted by $r$.

The Lennard-Jones potential is truncated at the cut-off radius $r_{\mathrm{c}}=2.5 \sigma$ and shifted. The parameters for the lubricant-lubricant interaction are those which have been reported for methane by Vrabec et al. [54] $\left(\varepsilon_{\mathrm{F}}=0.0151 \mathrm{eV}\right.$ and $\sigma_{\mathrm{F}}=$ $3.7241 \AA$ ). The lubricant has an initial density of $0.4389 \mathrm{~g} / \mathrm{cm}^{3}$.

The off-diagonal entries in Table 1 indicate the cross-interaction between sites of different substances, i.e. indenter-substrate, lubricant-substrate and indenterlubricant. The indenter-substrate interaction is purely repulsive. It is modeled by a Lennard-Jones potential [55], cut-off at the minimum of the potential [3,9,13], i.e. the Weaks-Chandler-Anderson potential (WCA) [56].

Lubricant sites interact with substrate and indenter sites in both lubricated scenarios in the same way via the LJTS potential. We refer to this as LJTS-wetting in Table 1 as the lubricant-substrate and lubricant-indenter adsorption energy determines the lubricants wetting state [50]. To study the influence of the wetting state on the indentation and scratching process, two different solid-fluid dispersion energies are considered in the present work: a weak solid-fluid interaction energy of $\varepsilon_{\text {weak-ads. }}=0.00503 \mathrm{eV}$ resulting in a contact angle of $\Theta=71^{\circ}$ and a stronger solid-fluid interaction energy of $\varepsilon_{\text {strong-ads. }}=0.01723 \mathrm{eV}$ resulting in a totally wetting state [50].

The size parameter for the solid-fluid interaction $\sigma_{\text {ads. }}$ is equal to that of the lubricant model. For the sake of brevity the two lubricated cases are denoted as weak- and strong adsorbing case in the following.

The simulations were performed with the open-source code LAMMPS [57]. The dislocation extraction algorithm (DXA) [58] is used to identify the dislocations, to determine their Burgers vectors, and to measure the total length of the dislocation lines $D$. The free software tools ParaView [59], VisIt [60] and OVITO [61] are employed to visualize the atomistic configurations.

\subsection{Definition of Observables}

Different observables are extracted from the simulation data and used for comparison of the three studied cases. The tangential force $F_{\mathrm{t}}$ and the normal force $F_{\mathrm{n}}$ on the indenter are evaluated as the sum of all pair interactions acting on the indenter in $x$ - and $z$-direction. The forces in $x$-direction are multiplied by 
-1 for convenience. They stem from the indenter-substrate and the indenter-fluid interactions.

$$
\begin{gathered}
F_{\mathrm{t}}=-\sum_{i=1}^{m}\left(F_{\mathrm{x} . i}^{\text {ind-subs }}+F_{\mathrm{x} . i}^{\text {ind-fluid }}\right) \\
F_{\mathrm{n}}=\sum_{i=1}^{m}\left(F_{\mathrm{y} \cdot i}^{\text {ind-subs }}+F_{\mathrm{y} \cdot i}^{\text {ind-fluid }}\right),
\end{gathered}
$$

where $m$ is the total number of atoms of the indenter. The COF is

$$
\mu=F_{\mathrm{t}} / F_{\mathrm{n}} .
$$

During indentation and scratching lattice defects are generated. They are quantified by the total dislocation length. The dislocations are identified using DXA [58]. Geometrical properties of the chip and the groove are determined using an alpha shape algorithm [62]. Thereby, the groove and the chip formation are recorded each 30 ps.

The gap between the indenter and the substrate is defined as indicated with the blue shaded area in Fig. 2 as the volume between the indenter and the substrate at $z<0$ and $x>x_{\mathrm{P}}$ (cf. Fig. 2), i.e. ahead of the indenter in the scratching direction. Both the gap volume and the number of trapped molecules are calculated from this definition. The contact between the indenter and the substrate can either be dry (no fluid molecules the gap) or lubricated (fluid molecules in the gap). Also the gap and chip volume is determined with the alpha shape algorithm.

We define a surface enlargement $A_{\text {real }} / A_{\text {ideal }}$ as the ratio of the real surface of the groove to its ideal surface, cf. Fig. 2. The real surface is again determined using the alpha shape algorithm. The ideal surface is

$$
A_{\text {ideal }}=2 \pi R d+2 x_{\mathrm{P}} R \arccos \left(\frac{R-d}{R}\right),
$$

where $R$ is the indenter radius, $d$ the indentation depth and $x_{\mathrm{P}}$ the scratching distance, cf. Fig. 2. Eq. (6) assumes a perfect spherical indenter and an ideal groove shape composed of a cylindrical and 2 semi-spherical sections. The surfacesite positions are employed to determine $A_{\text {real }}$. The particle size, i.e. size parameter $\sigma$, is not considered for $A_{\text {real }}$, which makes the surface enlargement independent from the employed force field and transferable to other problems.

Also the chip height $h_{\text {chip }}$ and volume $V_{\text {chip }}$ are calculated. The chip volume, as indicated in Fig. 2, is calculated from numerical integration of the chip surface area, which is also gained by the alpha shape algorithm. The reference for both is the surface of the unmachined substrate.

\section{Results and Discussion}

\subsection{Forces}

The total normal and total tangential force on the indenter $F_{\mathrm{n}}$ and $F_{\mathrm{t}}$ for all three cases (dry, weak- and strong adsorption) are compared in Fig. 3 (a and b). The average normal and tangential force $F_{\mathrm{n}}$ and $F_{\mathrm{t}}$ are zero in the first part of the 
indentation and in the last part of the retraction in which the indenter is not in contact with the substrate $(0-150 \mathrm{ps}$ and $750-860 \mathrm{ps})$. In the lubricated cases, $F_{\mathrm{n}}$ and $F_{\mathrm{t}}$ fluctuate around zero in these phases due to the presence of the lubricant. As shown in the insets in Fig. 3, the fluctuations in the case with strong adsorption are larger than in the case with weak adsorption, due to the weaker interactions.

During the indentation (125 - $300 \mathrm{ps}$ ) the normal force $F_{\mathrm{n}}$ rises strongly for all three cases. The rise starts earlier for the case with strong adsorption than for the case with weak adsorption due to the stronger influence of the adsorbed layers. Due to the absence of such layers, the rise of $F_{\mathrm{n}}$ starts later in the case without lubricant. There are two important dips in the curve for $F_{\mathrm{n}}$ for the case without lubricant which are due to the start of dislocation movements. This is a common observation, see e.g. [6]. Smaller dips are also present in the lubricated case with weak adsorption and, to a still lesser extent, in the case with strong adsorption. Hence, the hypothesis can be stated that the lubrication dampens the effects which lead to the start of the movement of dislocations.

Fig. $3 \mathrm{~b}$ ) shows the results for the tangential force $F_{\mathrm{t}}$. As long as the indenter is not in contact with the substrate, the observations are essentially the same as for $F_{\mathrm{n}}$ : there are small fluctuations around zero for the two lubricated cases due to the presence of the fluid. Once the indenter gets into contact with the substrate, the fluctuations increase and features of oscillations begin to develop for all three cases. These oscillations are clearly discernible for the dry case and the lubricated case with weak interaction, whereas they are damped for the case with strong interactions.

At the end of the indentation (300 ps), the values for $F_{\mathrm{n}}$ are similar for all three cases. For $F_{\mathrm{t}}$, they are essentially zero, not counting the deviation for the strongly adsorbing case which is a result of an asymmetric squeeze-out of fluid, as discussed below in more detail.

After the start of the lateral movement $(300-400 \mathrm{ps})$, the tangential force $F_{\mathrm{t}}$ increases rapidly as the chip starts to form, while the normal force $F_{\mathrm{n}}$ decreases as the contact area between the indenter and the substrate decreases. This decrease of contact area is due to the kinematics of the process, which forms a gap between the backside of the indenter and the substrate at the beginning of the lateral motion. This gap than quickly fills with fluid molecules in the lubricated cases as can be seen in Fig. 9. The increase of $F_{\mathrm{t}}$ is slower for the lubricated case with strong adsorption than for the other cases. This is a consequence of the fluid molecules that are trapped in the gap between the indenter and the substrate and are slowly squeezed out as described in more detail below.

After about $400 \mathrm{ps}$ a steady state with strong fluctuations is reached both for $F_{\mathrm{t}}$ and $F_{\mathrm{n}}$ in all three studied cases. The differences between these cases are within the band of the strong fluctuations. Again, oscillatory components are observed in the fluctuating signals for $F_{\mathrm{t}}$. During the retraction phase which starts at $700 \mathrm{ps}$ both $F_{\mathrm{n}}$ and $F_{\mathrm{t}}$ decrease to zero and there is no significant difference between the three cases.

The COF $\mu=F_{\mathrm{t}} / F_{\mathrm{n}}$ in the scratching phase is shown in Fig. 4-top for the three studied cases. Fig. 4 -bottom shows the difference $\Delta \mu$ between the two lubricated cases and the dry case, which is considered as the reference. The results for the three studied cases agree within the band of the strong fluctuations. A steady state is established after $100 \mathrm{ps}$ for which the COF is about $0.52 \pm 0.1$. 
The forces on the indenter result from contributions of the indenter-fluid and the indenter-substrate interactions, see Eq. (1) and (2). Fig. 5 shows these contributions to the normal force on the indenter $F_{\mathrm{n}}$. In the early phase of the indentation, the contribution of the indenter-fluid interaction dominates. Subsequently, its contribution to $F_{\mathrm{n}}$ declines and becomes minor in the stationary phase of the scratching phase. As expected, the contributions of the indenter-fluid interactions are more important for the case with strong adsorption than for the case with weak adsorption. Also the decline is slower for the case with strong adsorption, which is related to the slower squeeze-out of the liquid from the gap between the indenter and the substrate for that case. The corresponding results for the tangential force $F_{\mathrm{t}}$ are shown in Fig. 6 . They reveal that $F_{\mathrm{t}}$ is dominated by the indenter-substrate interactions.

\subsection{Dislocations}

During the indentation and scratching, dislocations are formed in the substrate. Fig. 7 shows the evolution of the dislocation network at different stages of the scratching process for the three cases. During the indentation and scratching process material is removed from the groove. This requires strong plastic deformation that shows up in the form of dislocations. The type of dislocations is identified by their Burgers vector $b$. For bcc Fe there are two important types of dislocations, those with $b=1 / 2<111>$ and those with $b=<100>$, see Fig. 7 . The first one is favored and dominates since it requires less energy to nucleate and move.

The formation of dislocations is characterized by the formation of long semiloops of $1 / 2<111>$ dislocations; these may even detach from the surface and move into the inner of the crystal as prismatic dislocation loops. During scratching the dislocation network changes by addition of newly formed dislocations as well as by the reaction of existing dislocations. The most prominent example of such a reaction is the merging of two $1 / 2<111>$ dislocations to form a $b=<100>$ dislocation. Note that the form of individual dislocations is subject to a high amount of stochastic randomness, caused by thermal fluctuations in the generation process. In view of these fluctuations, the dislocation patterns resulting under the influence of lubrication does not strongly differ from that in the dry contact. Thus, for instance, in all cases after removing the indenter the dominance of $b=1 / 2<$ $111>$ dislocations is reduced and $b=<100>$ dislocations become more dominant. This is caused by the high mobility of $b=1 / 2<111>$ dislocations that retract back to the surface during the removal of the indenter.

The processes of defect formation in the crystal can be quantitatively analyzed by the total dislocation length $D$, see Fig. 8, during the entire process of indentation, scratching, and retraction. Lubrication affects the first nucleation of dislocation in the indentation stage, where plasticity is generated earlier in the two lubricated cases than in the dry case. This appears evident from the fact that the lubricant can transfer forces from the indenter to the substrate already before the two solids are in direct contact. At the end of the indentation (300 ps), the total dislocation length is somewhat higher for the case with strong adsorption than in the other two cases. This can be understood as an effect of the adsorbed layers leading to an effective increase of the size of the indenter and thereby to more plastic deformation. During scratching, the total dislocation length increases 
in the dry case and in the case of the strongly adsorbing lubricant, while almost no increment for the case of weak adsorption lubrication is found. After the retraction, the total dislocation length decreases since some dislocations move to the surface and are annihilated there. During the scratching, the effect of the lubricant is hardly visible behind the stochastic fluctuations of the dislocation generation and reaction processes. The main effect of the lubrication on dislocation formation in the material lies in the earlier generation process during the indentation.

\subsection{Groove, Chip, and Contact Zone}

Fig. 9 shows screenshots of the contact zone of all three simulations (left: dry, center: weak adsorption, right: strong adsorption) at different simulation times. All indenter atoms are visible and opaque. Lubricant molecules are visible if they are underneath the unpenetrated substrate surface $z=0$ and substrate atoms are visible if they are above the unpenetrated substrate $z=0$. The differences between the three simulations are small but visible. The chip formation is similar in all cases until the end of the scratching $(700 \mathrm{ps})$. At this time the tip of the chip leans against the indenter in the dry case, while in the lubricated cases the tip of the chip is straight up. This is due to lubricant molecules between the tip of the chip and the indenter, especially in the strongly adsorbed case (not shown for clarity). The lubrication itself, i.e. the number of trapped molecules between the indenter and the substrate, differs between the strongly and weakly adsorption case. More molecules remain trapped in the strongly adsorption case, cf. $300 \mathrm{ps,}$ $390 \mathrm{ps}$ and $480 \mathrm{ps}$. But both cases have a dry contact zone (as defined in Fig. 1) after that, i.e. all lubricant molecules are squeezed out of the gap.

Fig. 10 shows the shape of the groove and chip formed. Most notable is the fact that dry and lubricated contact lead to the same form of groove and chip, although slight differences are present. The chip in the weakly adsorption case has a somewhat broader chip in $y$-direction than the other two cases.

The chip volume and maximum chip height is plotted in Fig. 11 a) and b) respectively. The chip volume increases in all three simulations steadily after the beginning of the lateral movement of the indenter. The increase continues at the beginning of the retraction $(700-750 \mathrm{ps})$, because the substrate relaxes elastically and thereby lifts the chip. While the three cases hardly differ in the chip volume at the beginning of the scratching (300-500 ps), they differ at the end of the scratching (500-700 ps). The chip volume of the strong and weak adsorption case is increased about $10 \%$ and $20 \%$ respectively compared to the dry case. This is mainly based on a mechanical coupling of the indenter and the chip, not only straight in front of the indenter where the chip is highest but also sideways in $y$-direction of the spherical indenter (cf. Fig. 10). In this flank of the groove the adsorbed lubricant molecules widen the indenter from the substrate point of view. The fact that the weakly adsorbed fluid leads to an even stronger increasing chip volume than the strongly adsorbed fluid is due to a better lateral mobility of lubricant molecules along the indenter and substrate surface $[63,64]$. This leads to a better inflow to the newly formed chip-indenter-fluid contact.

The same argument holds for the maximum chip height $h_{\text {chip }}$. It behaves similarly in between the three simulations until approximately 500 ps of the simulation time. Subsequently, the maximum chip height for the two lubricated cases lies 
about $10 \%$ above the corresponding dry simulation, although the two lubricated scenarios differ less in the chip height than the chip volume. The center of the chip is therefore less affected by the presence of a lubricant than the flanks of the chip and the flanks of the groove, cf. Fig. 10. The chip is mainly formed in the front of the groove, due to the scratch direction in $\left[\begin{array}{lll}0 & \overline{1} & \overline{1}\end{array}\right]$ and slip along $\left[\begin{array}{lll}1 & \overline{1} & \overline{1}\end{array}\right]$. This is a consequence of the slip systems activated in the single crystal: In bcc crystals, slip occurs on the $\{110\}$ - and to a lesser degree also $\{112\}$ planes - in the close-packed $<111>$ directions. Sideways slip is also generated, but it is not so prominent. This feature is typical for spherical indenters, but may change for other indenter shapes, see [3].

Fig. 12 shows the surface enlargement $A_{\text {real }} / A_{\text {ideal }}$ as defined in section 2.3 . A constant $A_{\text {real }} / A_{\text {ideal }}(t)$ implies a linearly increasing surface area, cf. Eq. (6). The surface enlargement differs strongly from unity at the beginning (300 - 400 ps) of the scratching and converges to a constant value slightly above unity. This is not only a result of the comparison of an atomically resolved surface with an ideal one, but also due to the definition of $A_{\text {ideal }}$, which does not take the atom's size into account, e.g. $\sigma_{\text {Ind.-Subs. }}$ The decay of $A_{\text {real }} / A_{\text {ideal }}$ at the beginning of the scratching $(300-500 \mathrm{ps})$ is a result of the elastic relaxation of the spherical part of the groove that the indenter leaves behind. The slope of $A_{\text {real }} / A_{\text {ideal }}(t)$ is similar in all three simulations, although throughout the scratching process the strongly absorbed fluid case has a higher surface enlargement than in the dry case. The weakly adsorbed fluid case does not differ significantly from the dry case. The surface enlargement lies in the strongly adsorbed case in average 0.08 above the dry one. This is due to a stamping of single or clustered lubricant molecules into the substrate, during the squeeze-out of lubricant molecules out of the contact zone underneath and aside the indenter. This mechanism was already described by Bhushan et al. [65]. At the solid-liquid interaction energies investigated in this study, the lubricant molecules are individually stamped into the substrate. No covering film is formed. This is shown in Fig. 13 comparing the dry case with the strong adsorbing lubricated case. Note how the inclusion of a lubricant renders the machined surface rougher (comparing Fig. 13a and b). The incorporation of lubricant atoms within the outermost atom layer of the workpiece, Fig. 13c and $\mathrm{d}$, represents the first step of forming a chemically transformed layer, which is denoted as tribolayer in the case that chemically reactive species are employed [66].

The development of the tip-substrate gap volume (as defined in Fig. 2) is shown in Fig. 14 a). The gap volume is only evaluated for those time steps when the bottom of the indenter has reached $z=0$. Since the gap volume is only calculated underneath the unpenetrated surface $z=0$, the gap volume increases linearly during the indentation. The gap volume remains fairly constant during the scratching. At the beginning of the retraction $(700-750 \mathrm{ps})$ the gap volume stays further constant, because the substrate follows the retraction movement of the indenter due to the substrate's elastic relaxation. After the substrate reaches its final plastically deformed groove, the gap volume increases again linearly. The gap volume at $z<0$ is hardly affected by the presence of a lubricant. However, the difference plot to the dry case in Fig. 14b) reveals that the strongly adsorbed fluid leads to a slightly increased gap volume, which is due to individually stamped molecules into the substrate, cf. Fig. 13. 
The number of trapped lubricant molecules in the gap between the indenter and the substrate (as defined in Fig. 2) during the contact process is shown in Fig. 14 c). Also this evaluation is only applied for those time steps, after the bottom edge of the indenter reaches the ground level of the substrate. The amount of trapped lubricant molecules increases in the strongly adsorbed fluid case during the indentation, as the lubrication gap is formed. On the contrary the number of lubricant molecules in the weakly adsorbed fluid case monotonously decreases already during the formation of the lubrication gap (180 - 300 ps).

The stronger adsorbed fluid remains longer attached to the indenter and the substrate surface, resulting in a slower squeeze-out. The lubricant molecules are squeezed out completely between 450 to $550 \mathrm{ps}$ during the scratching, which is in perfect agreement with the indenter-fluid force converging to zero at this simulation time (cf. Fig. 6). The weakly adsorbed fluid on the other side is already partially squeezed out during the indentation and completely pushed out of the gap until circa $520 \mathrm{ps}$, which is also in very good agreement with the indenter-fluid force, cf. Fig. 6. The number of trapped lubricant molecules is in the strongly adsorbed case in average about four times the amount in the weakly adsorbed case. The squeeze-out duration time is on the other side hardly affected by the adsorption interaction energy.

The molecule size $\sigma_{\mathrm{F}}$ has the same order of magnitude as the size parameter of the indenter-substrate interaction. Thus, the fluid molecules fit in the gap between the substrate and the indenter. Vice versa lubricant molecules only form a monolayer in the gap at the beginning of the contact process before they are squeezed out (cf. Fig. 9).

Furthermore, an interesting mechanism can be observed during the retraction of the indenter $(750-820 \mathrm{ps})$, when the amount of lubricant molecules in the gap increases, since the gap itself increases. Liquid fills this increasing gap. As already mentioned above, the lateral mobility of adsorbed molecules on a wall increases with a decreasing adsorption interaction energy [64,63]. Therefore lubricant molecules fill the gap faster during the retraction in the weakly adsorbed case, which is also clearly visible in the last screenshot in Fig. 9.

\section{Conclusions}

Nanoindentation and -scratching in a Fe (100) surface of a dry and lubricated contact process are studied by molecular dynamics simulation. The lubricant is modeled as a Lennard-Jones fluid. Two lubricated scenarios, differing in the solidfluid interaction energy, are compared with a dry one. While the lubricated case with a relatively weak adsorption energy behaves rather similar to the dry case, considerable differences are found between the contact process with a stronger adsorbing fluid compared to the dry case.

We find that the lubricant reduces fluctuations in the time evolution of most examined properties considerably, such as the friction forces, the total dislocation length and the chip formation, especially in the case of a strong adsorption energy. This reduction increases significantly with the adsorption energy. These effects are likely due to a mechanical coupling between the indenter and the substrate atoms via the lubricant which balance force peaks. 
The normal force acting on the tip during machining is only mildly affected by the presence and nature of the lubricant, while the tangential force is subject to strong fluctuations during the machining process. A closer look reveals that while the lubricant is still embedded between tip and substrate, the strongly adsorbing lubricant features an enhanced tangential force. We attribute this behavior to an effective enlargement of the tip size by the lubricant.

The lubricant forms a monolayer in the gap in both investigated lubricated cases but is squeezed-out of the contact zone quickly during the scratching. The amount of trapped lubricant molecules in the gap correlates with the total normal force between the indenter and the lubricant and increases significantly with the adsorption energy of lubricant molecules. The squeeze-out time is hardly affected and is around $200 \mathrm{ps}$ of the scratching time. Afterwards the contact zone is dry, i.e. no lubricant molecules are left in the gap.

The chip formation is only slightly influenced by the presence of a lubricant. The chip height is less affected by the presence of a lubricant than its width. The chip volume between the strongly and weekly adsorbed fluid cases and the dry case increases by $10 \%$ and $20 \%$, respectively. In our opinion this is a result of the inflow of lubricant molecules in the constantly newly formed gap between the indenter and the substrate, where material is moved. These lubricant molecules act like a broadening of the indenter - during the indentation in the entire contact zone and during the scratching mainly sideways of the indenter - and thereby lead to an increased material removal. The decrease of lateral mobility with an increasing solid-fluid interaction energy becomes also very clear by the fluid inflow during the retraction of the indenter out of the substrate.

An increasing adsorption energy of a lubricant has two opposing effects: (1) the lubricant molecules stick stronger in the gap, since more energy is needed to break the adsorption bonds and squeeze them out of the gap between the indenter and the substrate. (2) A weaker adsorption energy on the other hand favors the lateral mobility of lubricant molecules along the indenter and substrate surface which results in a better inflow to the contact zone during retraction.

Future work will aim at modeling more complex lubricant fluids, such as oils, and include a study of the influence of the fluid environment on the energy balance in the machining zone. In addition, the question to what extent the roughness of substrate and tool surfaces might enable a continuous inclusion of the lubricant within the tip-substrate gap will be explored.

\section{Figures and Tables}




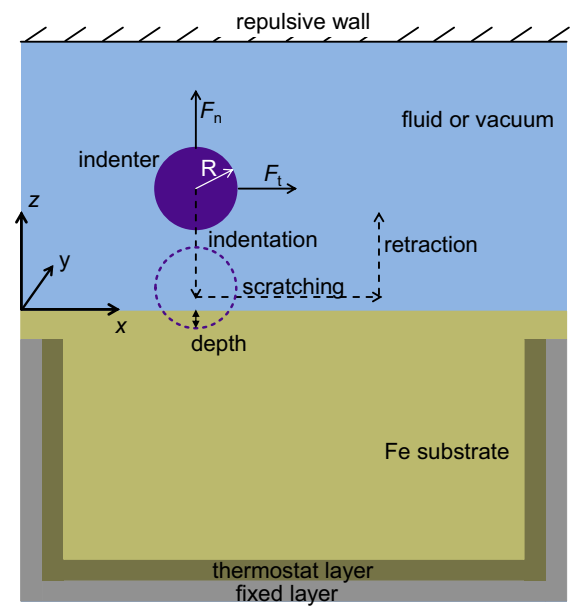

Fig. 1 Setup of the simulation box for the indentation, scratching and retraction of the indenter. The indenter is either in a vacuum environment (dry case) or immersed in fluid (two lubricated cases).

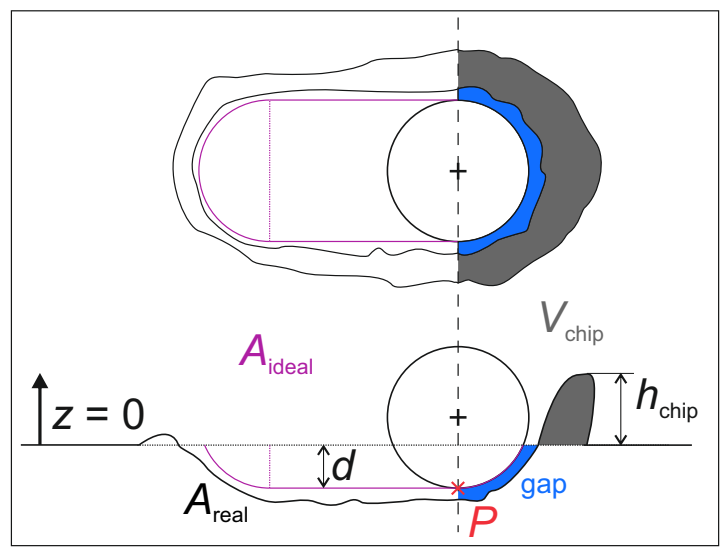

Fig. 2 Schematic definition of geometric parameters used for describing the studied process. The chip is characterized by its height and volume, the groove by the ratio of the ideal and the real groove surface. Blue indicates the lubrication gap presumed for the calculation of its volume and the lubricant molecules trapped in it. The point $P$ indicates the bottom point of the indenter. 


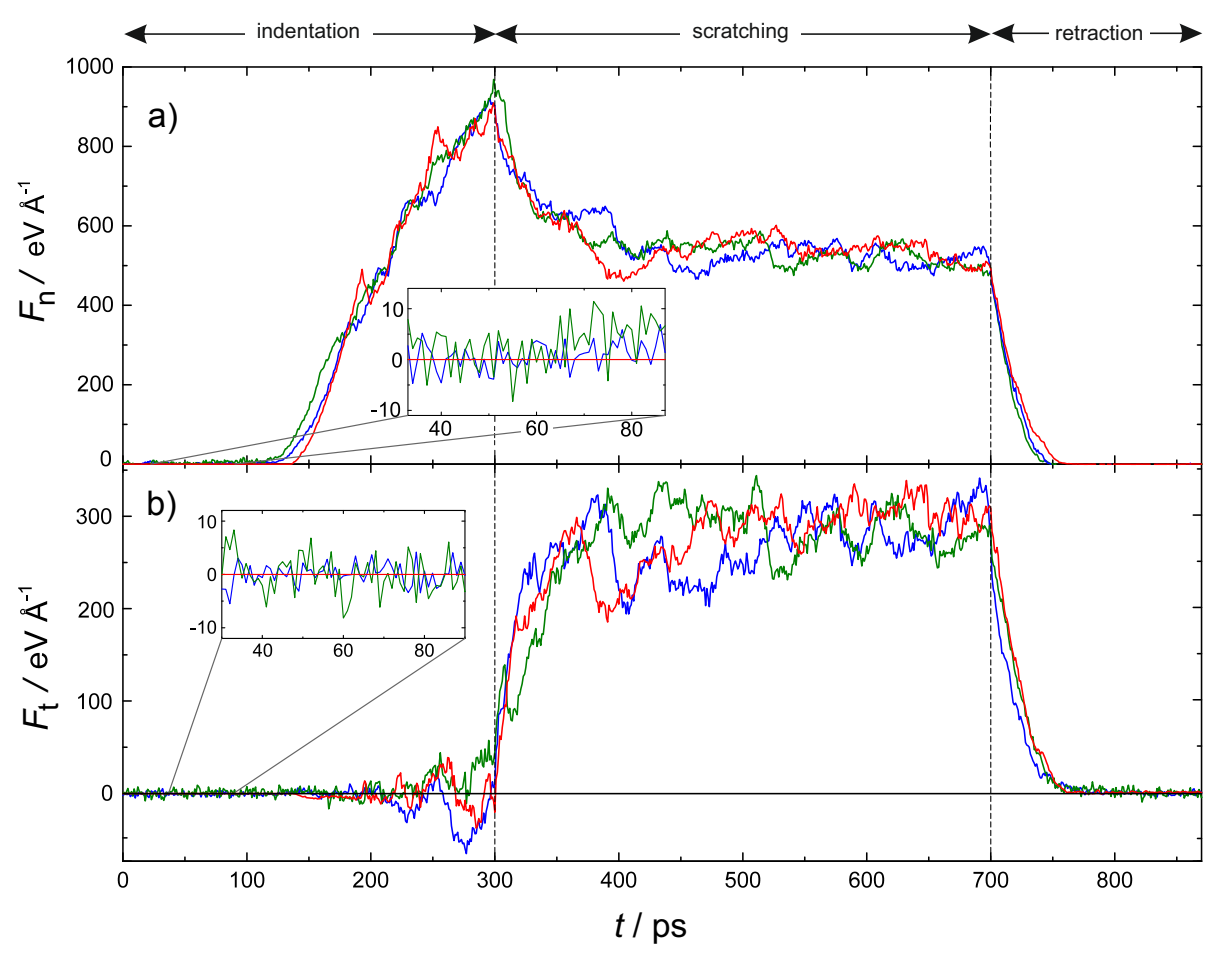

Fig. 3 Normal a) and tangential b) forces on the indenter during the entire simulation (indentation, scratching and retraction). The green and blue lines indicate the lubricated simulations, with strong and weak adsorption, respectively. The red line indicates the dry simulation.

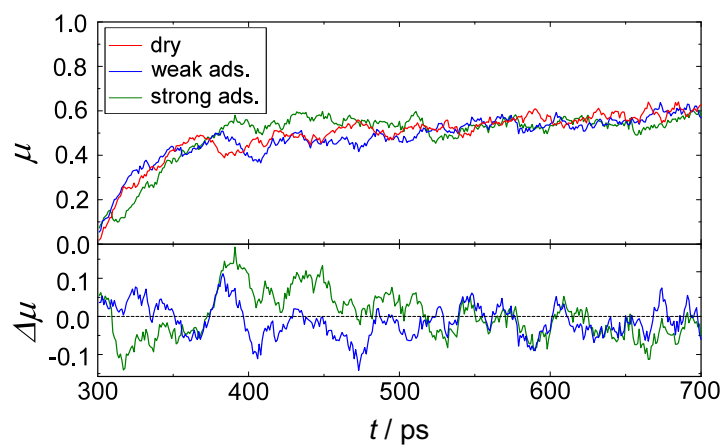

Fig. 4 Coefficient of friction $\mu$ during the scratching phase for the three studied cases (red: dry, blue weak adsorption, green: strong adsorption). In the lower part of the diagram the difference between the two lubriacted cases and the dry case is plotted. 


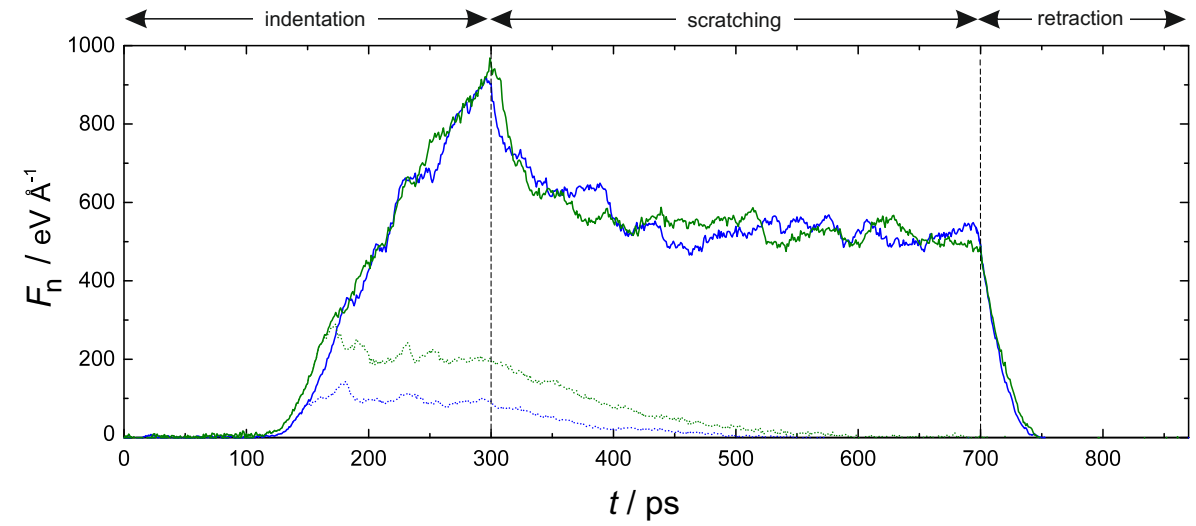

Fig. 5 Normal forces between the indenter and the fluid (dotted line) and the total forces on the indenter (solid line) during the simulation. The green and blue lines indicate the lubricated simulations, with strong and weak adsorption, respectively.

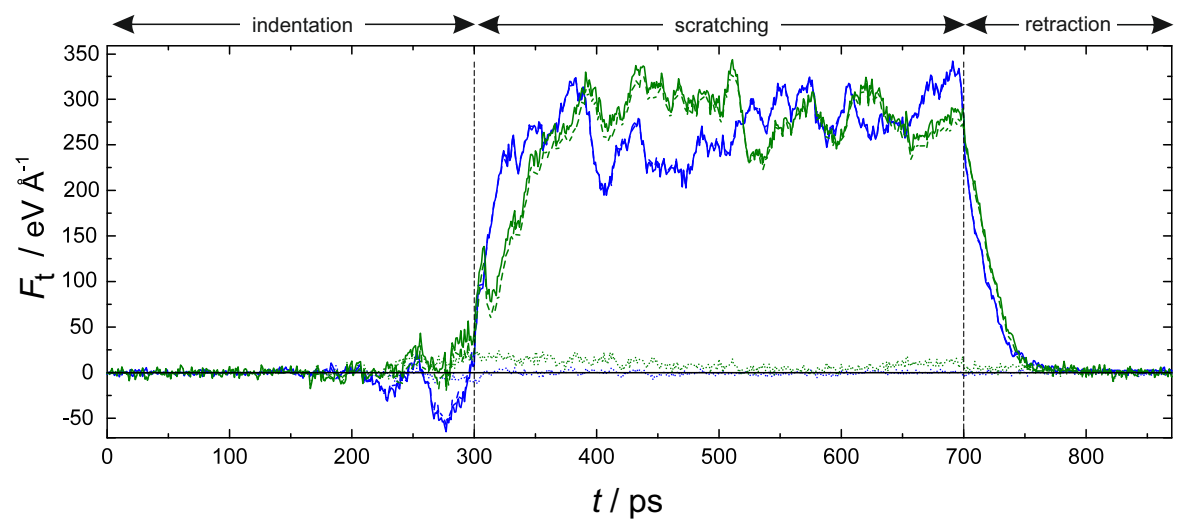

Fig. 6 Tangential forces between the indenter and the fluid (dotted line) and the total forces on the indenter (solid line) during the simulation. The green and blue lines indicate the lubricated simulations, with strong and weak adsorption, respectively. 


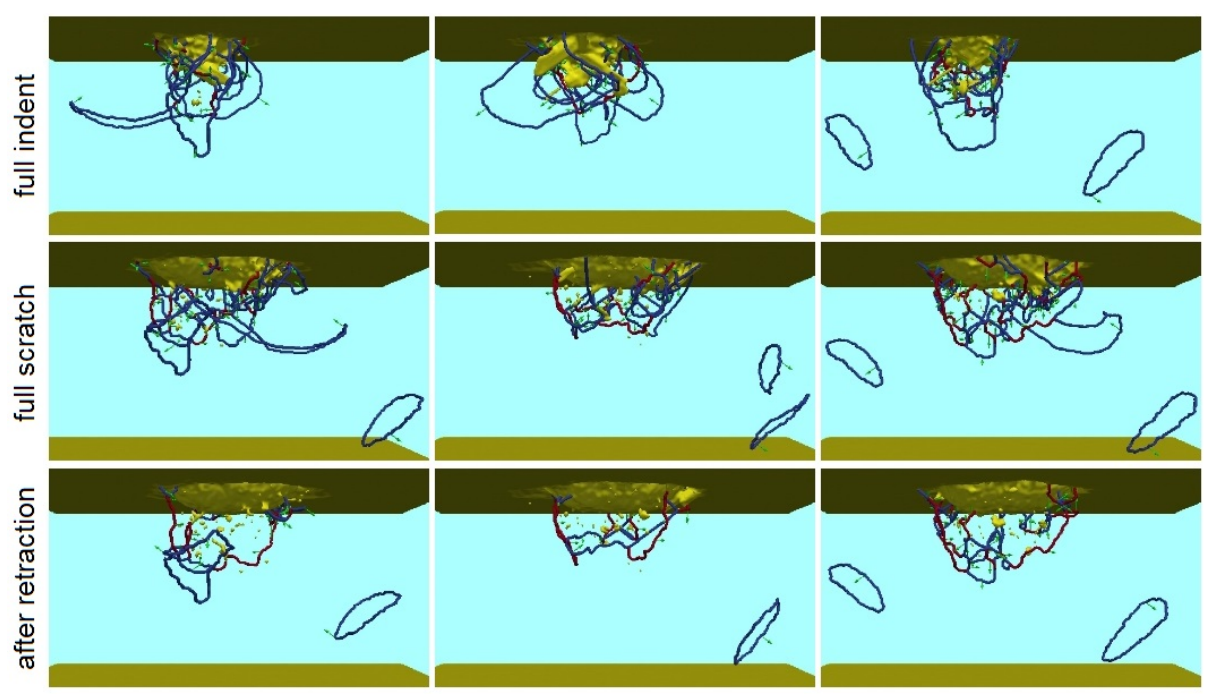

Fig. 7 Evolution of the dislocation network created in the penetrated substrate after full indentation (top row), at the end of the scratching (center row) and after the retraction of the indenter (bottom row). The case with the dry contact is shown in the left column; that of weak adsorption in the middle; and the case of strong adsorption is shown in the right column. Yellow indicates the deformed surface and point defects. Dislocation lines with Burgers vector $1 / 2<111>$ are shown in blue, those with $<100>$ in red. Green arrows indicate the direction of the Burgers vector.

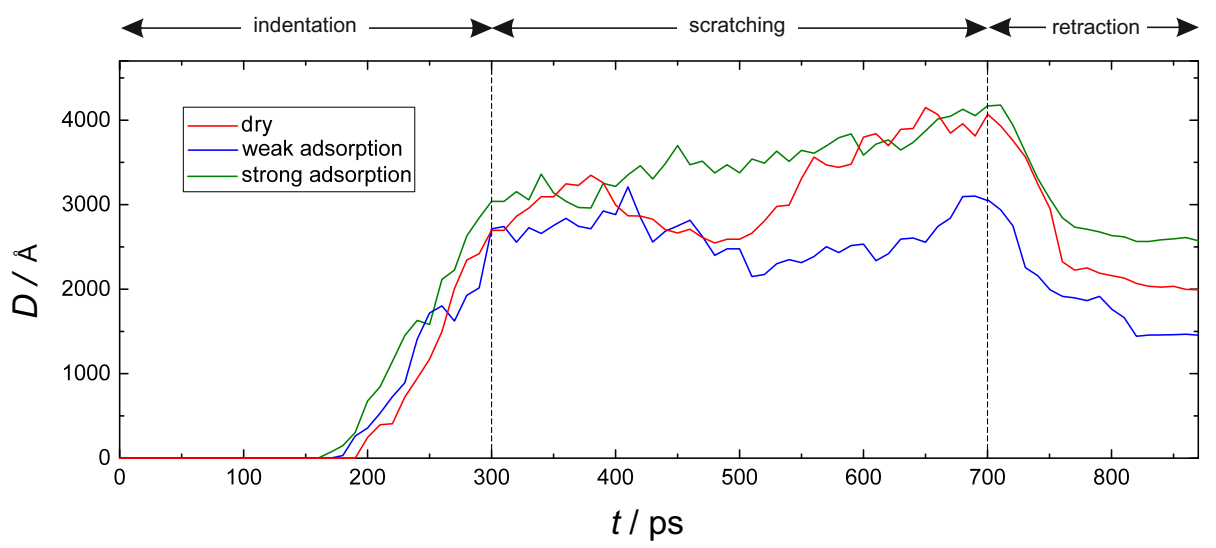

Fig. 8 Total dislocation length in the substrate during the simulation (indentation, scratching and retraction). The green and blue lines indicate the lubricated simulations, with strong and weak adsorption, respectively. The red line indicates the dry simulation. 

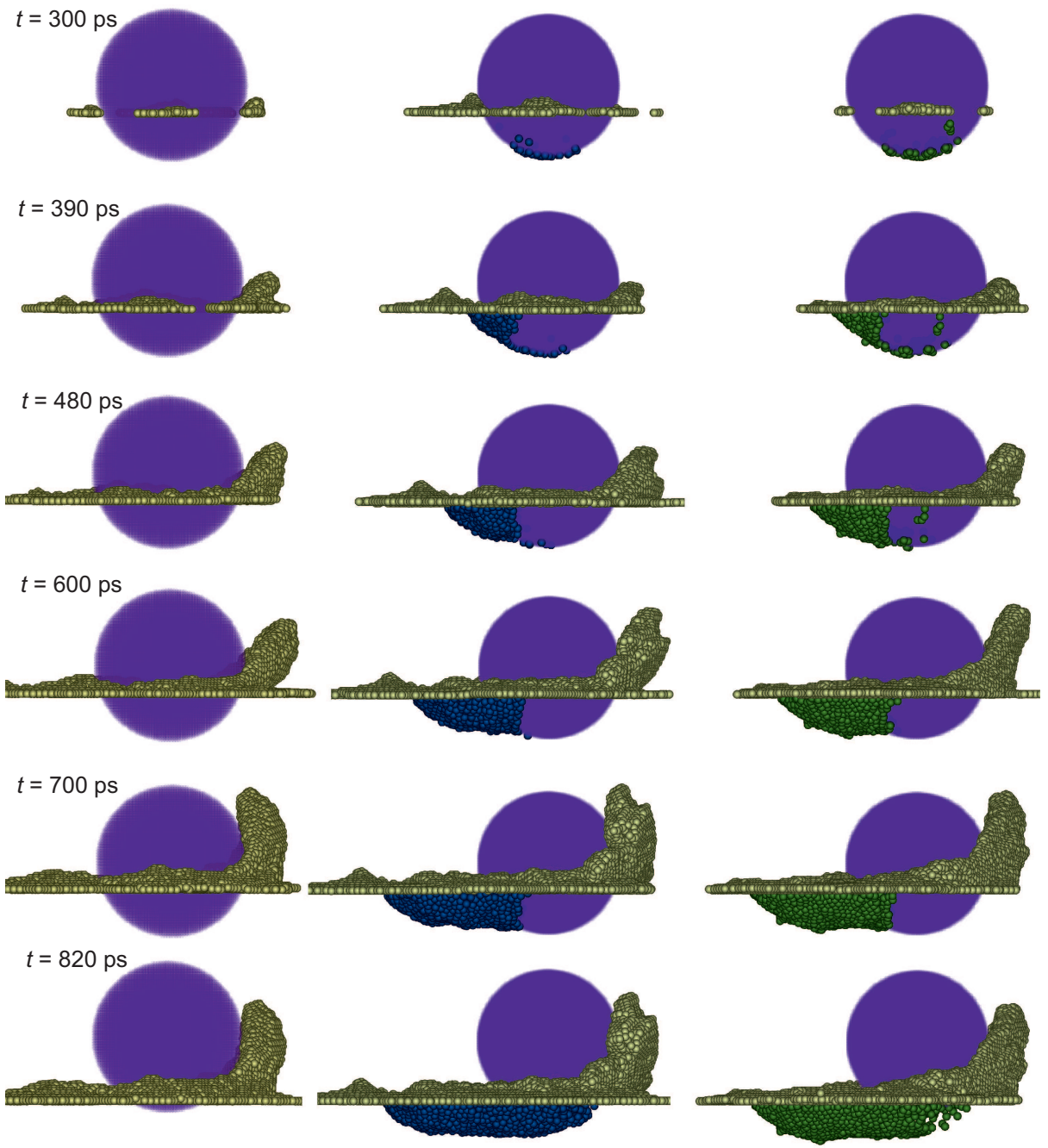

Fig. 9 Screenshots of the contact zone for all three simulations (left: dry; middle: weak adsorption; right: strong adsorption). Each row corresponds to the same simulation time. Only the substrate atoms with $z>0$ and the lubricant molecules with $z<0$ are visible for clarity. Yellow atoms indicate the substrate, blue and green the lubricant in the weak and strong adsorption case respectively. The spherical indenter is purple. 

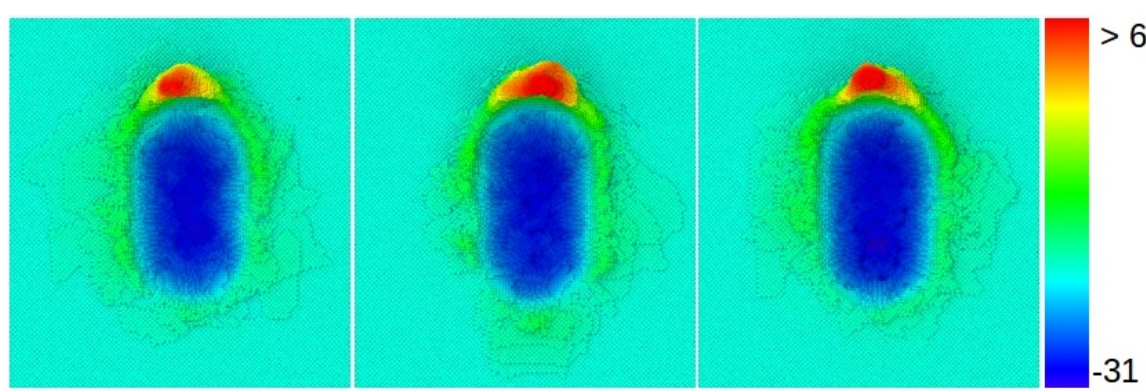

Fig. 10 Top view of the chip and groove formed after the retraction of the indenter for the case of a dry contact (left), weak adsorption (middle) and strong adsorption (right). The color indicates the distance to the reference plane with coordinate $z=0$. The absolute values are given in the legend on the right hand side.

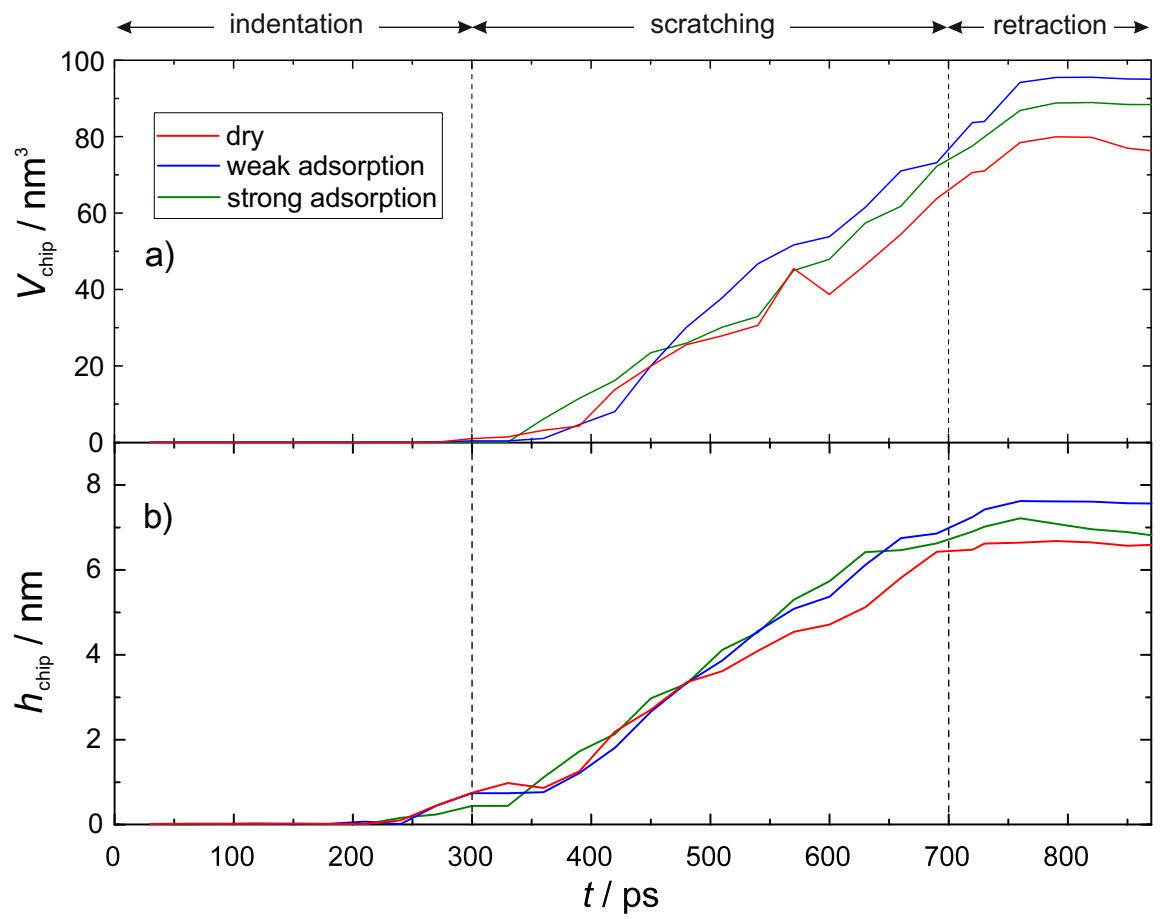

Fig. 11 Characterization of the chip evolution during the simulation (chip volume: a) and maximum chip height: b)). 


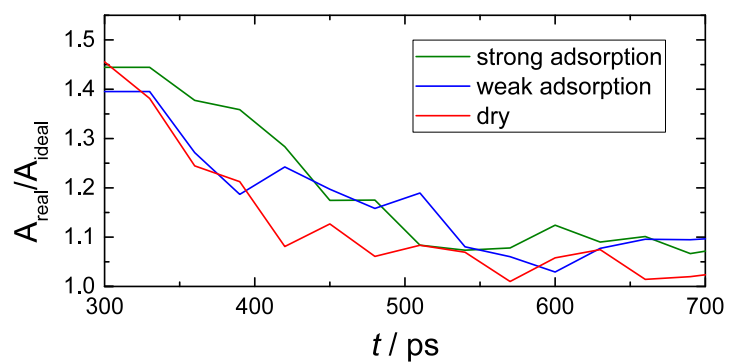

Fig. 12 Surface enlargement of the groove area, defined as the ratio of the real surface area $A_{\text {real }}$ of the groove in the simulation as sum of finite surface elements and the ideal area $A_{\text {ideal }}$, which is the groove of a perfect sphere scratched in a substrate.
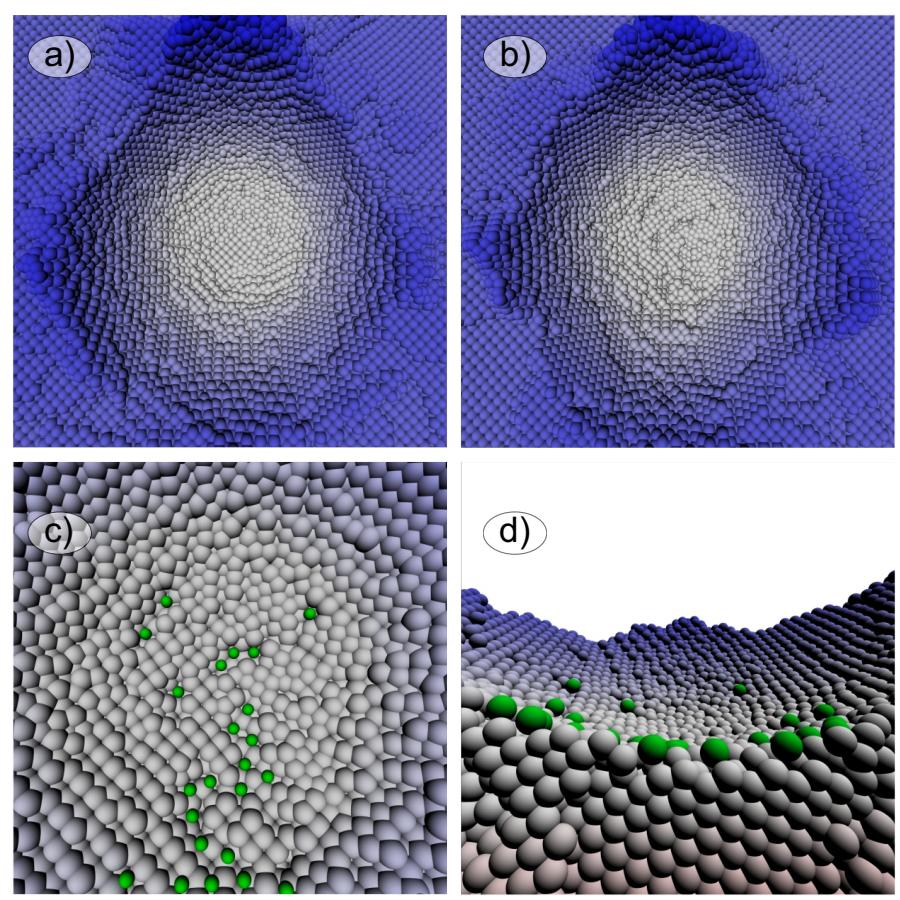

(d)

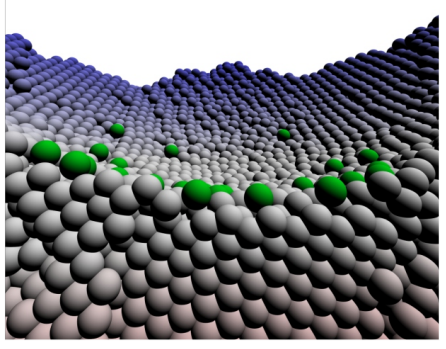

Fig. 13 Screenshots of the contact zone at a simulation time of $360 \mathrm{ps}$ from the dry case a) and the lubricated case with the strong adsorption energy b) c) and d). The indenter is in all pictures faded out; as is the lubricant in b). Grey and blue atoms indicate the substrate with the $z$-position as color code: grey at the bottom of the groove and blue at the top of the chip. Fig. a) and b) show the top view in negative $z$-direction. Fig. c) shows a closer zoom to the bottom of the groove from Fig. b). Fig. d) shows a cut view through a $x z$-plane at the bottom of the groove in positive $y$-direction. In both Fig. c) and d) the lubricant molecules are faded in and colored green. 


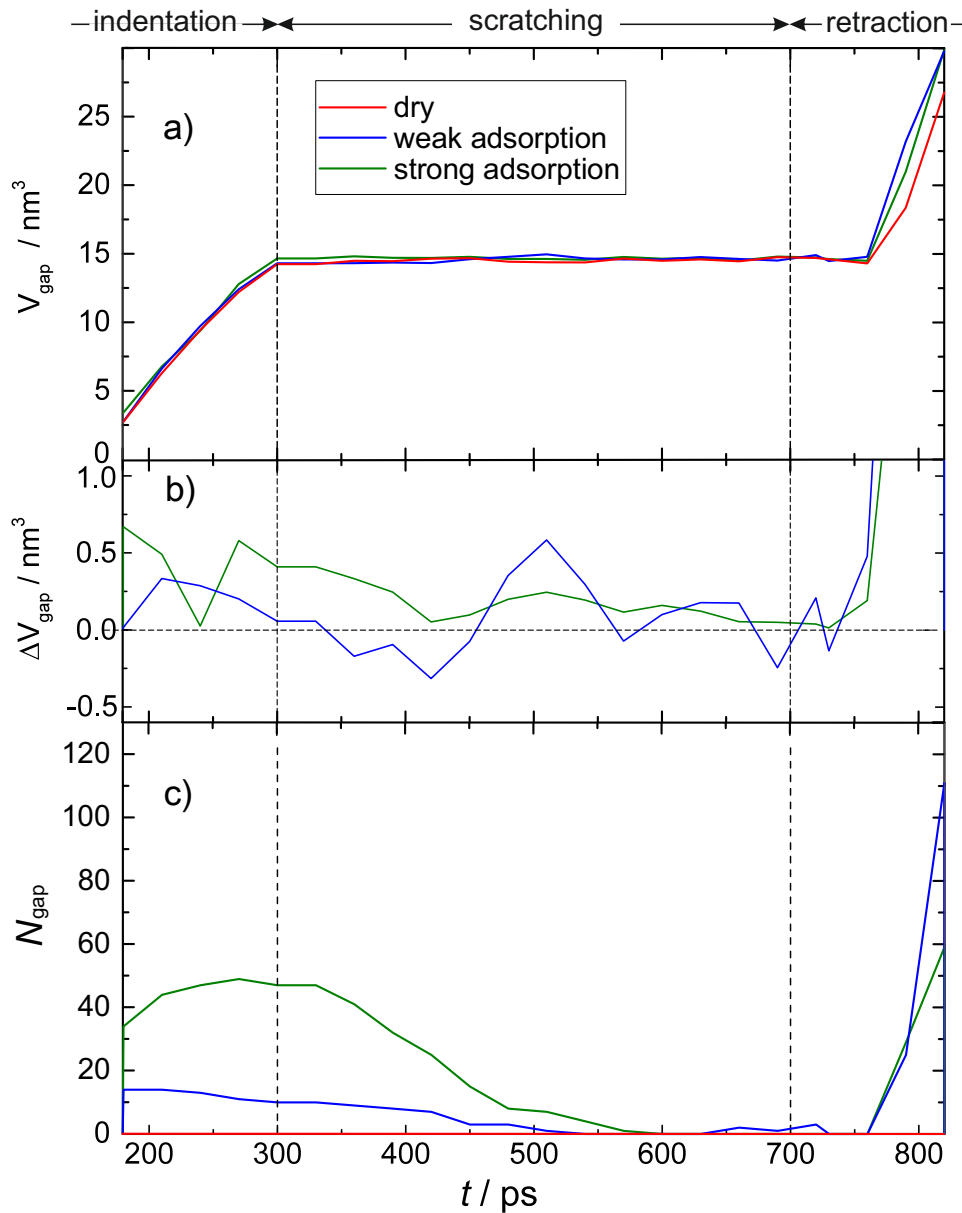

Fig. 14 a) shows the gap volume between the indenter and the substrate. b) shows the difference in the gap volume between the dry and the two lubricated cases, respectively. c) shows the number of trapped lubricant molecules in the gap (as defined in Fig. 2). 
Acknowledgements The authors gratefully acknowledge financial support by the DFG within IRTG 2057 Physical Modelling for Virtual Manufacturing Systems and Processes and CRC 926 Microscale Morphology of Component Surfaces. The simulations were carried out on the HAZELHEN at High Performance Computing Center Stuttgart (HLRS), on the ELWE at Regional University Computing Center Kaiserslautern (RHRK) under the grant TUKL-TLMV as well as on the SUPERMUC at Leibniz Supercomputing Centre (LRZ) Garching within the computing project SPARLAMPE (pr48te). The present research was conducted under the auspices of the Boltzmann-Zuse Society of Computational Molecular Engineering (BZS).

\section{References}

1. K. Maekawa, A. Itoh, Friction and tool wear in nano-scale machining - a molecular dynamics approach, Wear 188, 115 (1995)

2. R. Komanduri, N. Chandrasekaran, L. Raff, A review on the molecular dynamics simulation of machining at the atomic scale, Proceedings of the Institution of Mechanical Engineers B 215, 1639 (2001)

3. I. Alhafez, A. Brodyanski, M. Kopnarski, H. Urbassek, Influence of tip geometry on nanoscratching, Tribological Letters 65(26), 1 (2017)

4. Y. Gao, C. Lu, N. Huynh, G. Michal, H. Zhu, A. Tieu, Molecular dynamics simulation of effect of indenter shape on nanoscratching of Ni, Wear 267, 1998 (2009)

5. I. Alhafez, H. Urbassek, Scratching of hcp metals: A molecular-dynamics study, Computational Materials Science 113, 187 (2016)

6. Y. Gao, C. Ruestes, H. Urbassek, Nanoindentation and nanoscratching of iron: Atomistic simulation of dislocation generation and reactions, Computational Materials Science $\mathbf{9 0}$, $232(2014)$

7. C. Wu, T. Fang, J. Lin, Atomic-scale simulations of material behaviors and tribology properties for fcc and bcc metal films, Materials Letters 80, 59 (2012)

8. Y. Gao, C.J. Ruestes, D.R. Tramontina, H.M. Urbassek, Comparative simulation study of the structure of the plastic zone produced by nanoindentation, Journal of the Mechanics and Physics of Solids 75(Supplement C), 58 (2015). DOI https://doi.org/10.1016/j.jmps.2014.11.005

9. Y. Gao, H. Urbassek, Scratching of nanocrystalline metals: A molecular dynamics study of Fe, Applied Surface Science 389, 688 (2016)

10. L. Zhang, H. Zhao, Y. Yang, H. Huang, Z. Ma, M. Shao, Evaluation of repeated single-point diamond turning on the deformation behavior of monochrystalline silicon via molecular dynamics simulation, Applied Physics A (2014)

11. Y. Li, A. Goyal, A. Chernatynski, J. Jayashanker, M. Kautzky, S. Sinnott, S. Phillpot, Nanoindentation of gold and gold alloys by molecular dynamics simulations, Materials Science \& Engineering A 651, 346 (2016)

12. H. Aristizibal, P. Parra, P. Lpez, E. Restrepo-Parra, Atomistic-scale simulations of material behaviors and tribology properties for bcc metal films, Chin. Phys. B 25, 010204 (2016)

13. Y. Gao, A. Brodyanski, M. Kopnarski, H. Urbassek, Nanoscratching of iron: A molecular dynamics study of the influence of surface orientation and scratching direction, Computational Materials Science 103, 77 (2015)

14. J. Israelachvili, Intermolecular and Surface Forces, vol. Third Edition (Academic Press, San Diego, 2011)

15. I. Szlufarska, M. Chandross, R. Carpick, Recent advances in single-asperity nanotribology, Journal of Physics D: Applied Physics 41, 123001 (2008)

16. A. Vanossi, N. Manini, M. Urbakh, S. Zapperi, E. Tosatti, Modeling friction: From nanoscale to mesoscale, Reviews of Modern Physics 85, 512 (2013)

17. M. Müser, Theory and simulation of friction and lubrication, Lect. Notes Phys. 704, 65 (2006)

18. X. Zheng, H. Zhu, A. Tieu, B. Kosasih, A molecular dynamics simulation of 3d rough lubricated contact, Tribology International 67, 217 (2013)

19. X. Zheng, H. Zhu, B. Kosasih, A. Tieu, A molecular dynamics simulation of boundary lubrication: The effect of $n$-alkanes chain length and normal load, Wear 301, 62 (2013)

20. I. Sivebaek, B. Persson, The effect of surface nano-corrugation on the squeeze-out of molecular thin hydrocarbon films between curved surfaces with long range elasticity, Nanotechnology 27, 445401 (2016) 
21. J. Ren, J. Zhao, Z. Dong, P. Liu, Molecular dynamics study on the mechanism of afmbased nanoscratching process with water-layer lubrication, Applied Surface Science 346 , 84 (2015)

22. R. Chen, M. Liang, J. Luo, H. Lei, D. Guo, X. Hu, Comparison of surface damage under the dry and wet impact: Molecular dynamics simulation, Applied Surface Science 258, $1756(2011)$

23. C. Tang, L. Zhang, A molecular dynamics analysis of the mechanical effect of water on the deformation of silicon monocrystals subjected to nano-indentation, Nanotechnology 16, 15 (2005)

24. Y. Chen, H. Han, F. Fang, X. Hu, Md simulation of nanometric cutting of copper with and without water lubrication, Science China 57, 1154 (2014)

25. M. Chandross, C. Lorenz, M. Stevens, G. Grest, Simulations of nanotribology with realistic probe tip models, Langmuir 24, 1240 (2008)

26. R. An, L. Huang, Y. Long, B. Kalanyan, X. Lu, K. Gubbins, Liquid-soild nanofriction and interfacial wetting, Langmuir 32, 743 (2015)

27. J. Shi, Y. Zhang, K. Sun, L. Fang, Effect of water film on the plastic deformation of monocrystalline copper, RSC Advances 6, 96824 (2016)

28. Y. Jeng, P. Tsai, Y. Liu, Adsorbed multilayer effects on the mechanical properties in nanometer indentation depth, Materials Research Bulletin 44, 1995 (2009)

29. W. Lee, S. Ju, C. Cheng, A molecular dynamics study of nanoindentation on a methyl methacrylate ultrathin film on a au (111) substrate: Interface and thickness effects, Langmuir 24, 13440 (2008)

30. F. Yang, R. Carpick, D. Srolovitz, Mechanics of contact, adhesion and failure of metallic nanoasperites in the presence of adsorbates: Toward conductive contact design, ACS Nano 11, 490 (2017)

31. L. Dai, V. Sorkin, Y. Zhang, Effect of surface chemistry on the mechanics and governing laws of friction and wear, ACS Applied Materials \& Interfaces 8, 8765 (2016)

32. B. Shiari, R. Miller, D. Klug, Multiscale simulation of material removal processes at the nanoscale, Journal of the Mechanics and Physics of Solids 55, 2384 (2007)

33. C. Greiner, J. Felts, Z. Dai, R. King, W.P. andCarpick, Controlling nanoscale friction through th competition between capillary adsorption and thermally activated sliding, ACSNano 6(5), 4305 (2012)

34. S. O'Shea, N. Gosvami, L. Lim, W. Hofbauer, Liquid atomic force microscopy: Solvation forces, molecular order, and squeeze-out, Japanese Journal of Applied Physics 49, 08LA01 (2010)

35. E. Cihan, S. Ipek, M. Baykara, Structural lubricity under ambient conditions, Nature Communications 7, 12055 (2016)

36. R. Rentsch, I. Inasaki, Effects of fluids on the surface generation in material removal processes - molecular dynamics simulation -, Annals of the CIRP 55, 601604 (2006)

37. M. Lautenschlaeger, S. Stephan, H. Urbassek, B. Kirsch, J. Aurich, M. Horsch, H. Hasse, Effects of lubrication on the friction in nanometric machining processes: A molecular dynamics approach, Applied Mechanics and Materials 869, 85 (2017). DOI 10.4028/www.scientific.net/AMM.869.85

38. M. Lautenschlaeger, S. Stephan, M. Horsch, B. Kirsch, J. Aurich, H. Hasse, Effects of lubrication on the friction and heat transfer in machining processes on the nanoscale: A molecular dynamics approach, Procedia CRIP (2017)

39. U. Tartaglino, I. Sivebaek, B. Persson, E. Tosatti, Impact of molecular structure on the lubrication squeeze-out between curved surfaces with long range elasticity, The Journal of Chemical Physics 125, 014704 (2006)

40. M. Allen, D. Tildesley, Computer Simulation of Liquids (Oxford, 2009)

41. H. Hou, Y. Zhang, Z. Li, T. Jiang, J. Zhang, C. Xu, Numerical analysis of entropy production on a lng cryogenic submerged pump, Journal of Natural Gas Science and Engineering 36, 87 (2016). DOI https://doi.org/10.1016/j.jngse.2016.10.017

42. A. Bahadori, in Handbook of Liquefied Natural Gas, ed. by S. Mokhatab, J.Y. Mak, J.V. Valappil, D.A. Wood (Gulf Professional Publishing, Boston, 2014), pp. 147 - 183. DOI https://doi.org/10.1016/B978-0-12-404585-9.00003-9

43. R.C. Bill, D. Wisander, Recrystallization as a controlling process in the wear of some f.c.c. metals, Wear 41(2), 351 (1977). DOI https://doi.org/10.1016/0043-1648(77)90013-8

44. D.W. Wisander, Friction and wear of selected metals and alloys in sliding contact with aisi 440c stainless steel in liquid methane and in liquid natural gas, NASA technical rep. 1150, 1 (1978) 
45. T. Kanda, M. Sato, T. Kimura, H. Asakawa, Expander and coolant-bleed cycles of methane-fueled rocket engines, Transactions of the Japan Society for Aeronautical and space sciences 61(3), 106 (2018). DOI 10.2322/tjsass.61.106

46. J. Collins, E. Hurlbert, K. Romig, J. Melcher, A. Hobson, P. Eaton, Sea-Level Flight Demonstration and Altitude Characterization of a LO2/LCH4 Based Ascent Propulsion Lander, 45th AIAA/ASME/SAE/ASEE Joint Propulsion Conference proceedings 45, 4948 (2009). DOI 10.2514/6.2009-4948

47. F. Cao, J.D. Deetz, H. Sun, Free energy-based coarse-grained force field for binary mixtures of hydrocarbons, nitrogen, oxygen, and carbon dioxide, Journal of Chemical Information and Modeling 57(1), 50 (2017). DOI 10.1021/acs.jcim.6b00685. URL https://doi.org/10.1021/acs.jcim.6b00685. PMID: 28029243

48. E. Brinksmeier, J. Aurich, E. Goveka, C. Heinzel, H. Hoffmeister, F. Klocke, J. Peters, R. Rentsch, D. Stephenson, E. Uhlmann, K. Weinert, M. Wittmann, Advances in modeling and simulation of grinding processes, Annals of the CIRP 55, 667 (2006)

49. Y. Yildiz, M. Nalbant, A review of cryogenic cooling in machining processes, International Journal of Machine Tools and Manufacture 48(9), 947 (2008). DOI https://doi.org/10.1016/j.ijmachtools.2008.01.008. URL http://www.sciencedirect.com/science/article/pii/S0890695508000242

50. S. Becker, H. Urbassek, M. Horsch, H. Hasse, Contact angle of sessile drops in lennardjones systems, Langmuir 30, 13606 (2014)

51. S. Becker, M. Kohns, H.M. Urbassek, M. Horsch, H. Hasse, Static and dynamic wetting behavior of drops on impregnated structured walls by molecular dynamics simulation, The Journal of Physical Chemistry C 121(23), 12669 (2017). DOI 10.1021/acs.jpcc.6b12741

52. H.J.C. Berendsen, J.P.M. Postma, W.F. van Gunsteren, A. DiNola, J.R. Haak, Molecular dynamics with coupling to an external bath, The Journal of Chemical Physics 81(8), 3684 (1984). DOI 10.1063/1.448118

53. M.I. Mendelev, S. Han, D.J. Srolovitz, G.J. Ackland, D.Y. Sun, M. Asta, Development of new interatomic potentials appropriate for crystalline and liquid iron, Philosophical Magazine 83(35), 3977 (2003). DOI 10.1080/14786430310001613264

54. J. Vrabec, G.K. Kedia, G. Fuchs, H. Hasse, Comprehensive study of the vapour-liquid coexistence of the truncated and shifted Lennard-Jones fluid including planar and spherical interface properties, Molecular Physics 104, 1509 (2006)

55. S. Banerjee, S. Naha, I.K. Puri, Molecular simulation of the carbon nanotube growth mode during catalytic synthesis, Applied Physics Letters 92(23), 233121 (2008). DOI 10.1063/1.2945798

56. J.D. Weeks, D. Chandler, H.C. Andersen, Role of repulsive forces in determining the equilibrium structure of simple liquids, The Journal of Chemical Physics 54(12), 5237 (1971). DOI 10.1063/1.1674820

57. S. Plimpton, Fast parallel algorithms for short-range molecular dynamics, J. Comp. Phys. 117, 1 (1995). URL http://lammps.sandia.gov

58. A. Stukowski, K. Albe, Extracting dislocations and non-dislocation crystal defects from atomistic simulation data, Modelling and Simulation in Materials Science and Engineering 18(8), 085001 (2010). DOI 10.1088/0965-0393/18/1/015012. URL http://www.ovito.org/

59. A. Henderson. Paraview guide, a parallel visualization application. Kitware Inc. (2007). URL http://www.paraview.org. Http://www.paraview.org

60. H. Childs, E. Brugger, B. Whitlock, J. Meredith, S. Ahern, D. Pugmire, K. Biagas, M. Miller, C. Harrison, G.H. Weber, H. Krishnan, T. Fogal, A. Sanderson, C. Garth, E.W. Bethel, D. Camp, O. Rübel, M. Durant, J.M. Favre, P. Navrátil, in High Performance Visualization-Enabling Extreme-Scale Scientific Insight (2012), pp. 357-372

61. A. Stukowski, Visualization and analysis of atomistic simulation data with OVITO - the open visualization tool, Modelling and Simulation in Materials Science and Engineering 18(1), 015012 (2010). DOI 10.1088/0965-0393/18/1/015012. URL http://www.ovito.org/. Http://www.ovito.org/

62. H. Edelsbrunner, D. Kirkpatrick, R. Seidel, On the shape of a set of points in the plane, IEEE Transactions on Information Theory 29(4), 551 (1983). DOI 10.1109/TIT.1983.1056714

63. X. Yong, L.T. Zhang, Slip in nanoscale shear flow: Mechanisms of interfacial friction, Microfluidics and Nanofluidics 14(1-2), 299 (2013). DOI 10.1007/s10404-012-1048-x

64. P.A. Thompson, A general boundary condition for liquid flow at solid surfaces, nature 389, 360 (1997) 
65. B. Bhushan, J. Israelachvili, U. Landman, Nanotribology: Friction, wear and lubrication at the atomic scale, Nature 374, 607 (1995)

66. P. Stoyanov, R. Merz, P. Romero, F.C. Whlisch, O.T. Abad, R. Gralla, P. Stemmer, M. Kopnarski, M. Moseler, R. Bennewitz, M. Dienwiebel, Surface softening in metal ceramic sliding contacts: an experimental and numerical investigation, American Chemical Society Nano 9, 1478 (2015) 
Table 1 Interaction types employed in the present study.

\begin{tabular}{r|rrr} 
& substrate & indenter & lubricant \\
\hline substrate & EAM $^{(1)}$ & WCA $^{(2)}$ & LJTS-wetting $^{(4)}$ \\
indenter & - & rigid body & LJTS-wetting $^{(4)}$ \\
lubricant & - & - & LJTS-lubricant
\end{tabular}

${ }^{(1)}$ embedded atom model [53]; ${ }^{(2)}$ Weaks-Chandler-Anderson potential [56] ; ${ }^{(3)}$ Lennard-Jones truncated and shifted fluid [54]; ${ }^{(4)}$ wetting behavior of LJTS-fluid [50]. 